\title{
Blackcurrant seed press residue increases tocopherol concentrations in serum and stool whilst biomarkers in stool and urine indicate increased oxidative stress in human subjects
}

\author{
Dorit Helbig ${ }^{1}$, Andreas Wagner ${ }^{1}$, Michael Glei ${ }^{1}$, Samar Basu ${ }^{2}$, Rainer Schubert ${ }^{1}$ and Gerhard Jahreis ${ }^{1}$ * \\ ${ }^{1}$ Department of Nutritional Physiology, Friedrich Schiller University of Jena, Dornburger Strasse 24-29, D-07743 Jena, Germany \\ ${ }^{2}$ Division of Clinical Nutrition and Metabolism, Department of Public Health and Caring Sciences, Uppsala University, Uppsala \\ Science Park, 75185 Uppsala, Sweden
}

(Received 4 September 2008 - Revised 19 November 2008 - Accepted 5 December 2008 - First published online 23 March 2009)

Berry seeds are a tocopherol-rich by-product of fruit processing without specific commercial value. In a human intervention study, the physiological impact of blackcurrant seed press residue (PR) was tested. Thirty-six women (aged $24 \pm 3$ years; twenty non-smokers, sixteen smokers) consumed $250 \mathrm{~g}$ bread/d containing $8 \%$ PR for a period of 4 weeks (period 3). Comparatively, a control bread without PR (250 g/d) was tested (period 2) and baseline data were obtained (period 1). Blood, stool and $24 \mathrm{~h}$ urine were collected during a $5 \mathrm{~d}$ standardised diet within each period. Tocopherol and Fe intakes were calculated from food intake. In serum, tocopherol concentration and Fe parameters were determined. In urine, oxidative stress markers 8-oxo-2'-deoxyguanosine, 8-iso- $\mathrm{PGF}_{2 \alpha}$ and inflammatory response marker 15 -keto-dihydro-PGF $2 \alpha$ were analysed. Stool tocopherol concentration, genotoxicity of faecal water (comet assay) and antioxidant capacity of stool (aromatic hydroxylation of salicylic acid) were determined. Fe and total tocopherol intake, total tocopherol concentrations in serum and stool, and genotoxicity of faecal water increased with PR bread consumption $(P<0.05)$. The antioxidant capacity of stool decreased between baseline and intervention, expressed by increased formation of 2,3- and 2,5-dihydroxybenzoic acid in vitro $(P<0 \cdot 05)$. In smokers, 8-oxo-2'-deoxyguanosine increased with $\mathrm{PR}$ consumption $(P<0.05)$. Prostane concentrations were unaffected by PR bread consumption. In summary, the intake of bread containing blackcurrant PR for 4 weeks increased serum and stool total tocopherol concentrations. However, various biomarkers indicated increased oxidative stress, suggesting that consumption of ground berry seed may not be of advantage.

Blackcurrants: Tocopherol: Comet assay: Antioxidant capacity in stool

Berry seeds, a by-product of juice fabrication with promising health benefits, are currently an unutilised nutritional resource. The seeds are rich in PUFA, tocopherols, fibre and contain polyphenols $^{(1,2)}$. Most of these ingredients are known to be associated with oxidation processes.

Oxidation of cell compounds such as DNA, lipids or proteins is described as a promotion of atherogenesis and carcinogenesis $^{(3,4)}$. Evidence for the correlation between oxidative DNA damage and human degenerative diseases such as CHD has previously been provided ${ }^{(5)}$. It is presumed that the prevalence of $\mathrm{CHD}$ and also of carcinogenesis is associated with tocopherol in serum or with tocopherol intake, although results are ambivalent. However, it has been shown that CHD patients are characterised by a high serum $\alpha$-: $\gamma$-tocopherol ratio ${ }^{(6)}$ and decreased $\gamma$-tocopherol serum concentration compared with controls ${ }^{(7)}$. In addition, the deficiency of $\alpha$-tocopherol in lipoproteins was not associated with atherosclerosis ${ }^{(8)}$. The results of the few studies dealing with fruit or berry consumption show inconclusive evidence of the effects on DNA damage in leucocytes ${ }^{(9)}$.
The findings of the Nurses' Health Study deny an association of vitamin E supplementation and the risk of colon cancer ${ }^{(10)}$.

Studies concerning berry consumption basically focused on whole berries or berry juices and extracts, rather than on the seeds and their potential ${ }^{(11-13)}$. Thus, the present human intervention study was conducted in order to correct omission. Different markers were used to evaluate the physiological and health-beneficial impact of blackcurrant press residue (PR). The most likely effects are probably due to the antioxidant potential of substances in PR. The matrices stool, urine and serum were included. Stool is a very complex and individual matrix, with various substance groups that may affect the antioxidant capacity. Examining one single substance is not always consequential, since synergies and correlations between different substances are very likely. The influence of PR on antioxidant capacity of stool can be evaluated by means of the aromatic hydroxylation of salicylic acid ${ }^{(14)}$. Characterising the DNA-damaging potential in stool can be performed using microgel electrophoresis with faecal waterincubated cells ${ }^{(15,16)}$. Genotoxicity of faecal water indicates

Abbreviations: DHBA, dihydroxy benzoic acid; 8-oxodG, 8-oxo-2'-deoxyguanosine; PI, period 1; PII, period 2; PIII, period 3; PR, press residue. 
the exposition of colon cells towards genotoxic compounds that lead to increased DNA damage and a risk of colon cancer.

Further, in the present study the influence of PR on oxidative DNA damage of the whole body was measured using the biomarker 8-oxo- $2^{\prime}$-deoxyguanosine (8-oxodG) in urine. 8-OxodG, a product of DNA repair excreted in urine, is generated by oxidative stress causing the transformation of the nucleoside guanosine ${ }^{(17)}$. The excreted amount of 8 -oxodG correlates with the extent of oxidative stress in an individual. Oxidative stress occurs as a result of different factors such as the presence of disease ${ }^{(18)}$, smoking ${ }^{(19)}$, physical exercise in untrained subjects ${ }^{(20)}$ and enzymic activities such as that of glutathione-S-transferase ${ }^{(21)}$. On the other hand, there are factors that can contribute to decrease the oxidative stress. These factors include a high consumption of fruits and vegetables containing significant concentrations of polyphenols and other substances ${ }^{(22)}$, moderate physical activity ${ }^{(23)}$ or the use of hormonal contraceptives ${ }^{(24)}$.

The aim of the study was to test whether serum total tocopherol concentrations and parameters linked to oxidative stress are influenced by the intake of blackcurrant PR in human subjects. Secondarily, effects of lifestyle parameters that are associated with oxidative stress such as smoking or the use of hormonal contraceptives were also considered.

\section{Experimental methods}

\section{Test substance, diets and experimental design}

Berry seed PR contains significant amounts of tocopherols $(\alpha, 6.56 \mu \mathrm{mol} / 100 \mathrm{~g} ; \beta, 0.32 \mu \mathrm{mol} / 100 \mathrm{~g} ; \gamma, 14.3 \mu \mathrm{mol} / 100 \mathrm{~g}$; $\delta, 0.44 \mu \mathrm{mol} / 100 \mathrm{~g})$ as well as $\gamma$-tocotrienol $(0.29 \mu \mathrm{mol} /$ $100 \mathrm{~g}$ ) according to Helbig et al. ${ }^{(1)}$. The PR has a gallic acid equivalent of $0.17 \mathrm{~g} / 100 \mathrm{~g}$, and a trolox equivalent of $2.82 \mathrm{mmol} / 100 \mathrm{~g}$ in a hydrophilic- and $67.2 \mu \mathrm{mol} / 100 \mathrm{~g}$ in a lipophilic solvent. As described previously, the PR contains $25.7 \mathrm{~g}$ fat, $48.2 \mathrm{~g}$ fibre, $22.5 \mathrm{~g}$ crude protein and $19 \mathrm{mg} \mathrm{Fe} / 100 \mathrm{~g}^{(1)}$. Inositol hexaphosphate (phytic acid) concentration of the applied PR was $0.63 \mathrm{mmol} / 100 \mathrm{~g}$ and no intermediate inositol phosphates were quantified. $\beta$-Carotene and plant sterols were not present in significant amounts ${ }^{(1)}$. The concentrations of yeasts, mould and bacteria measured (D Helbig, unpublished results) were all below the upper levels considered safe for ground grain products with reference to the values released by the German Society of Hygiene and Microbiology (Regulation (EU) 2073/2005).

Blackcurrant seeds were pressed, ground and sieved to obtain an acceptable mouth feeling. The PR was baked into bread at a maximum dose of $8 \%$ that still allowed the correct preparation of the dough. No significant losses of tocopherol were verified by the baking process. The daily amount of $250 \mathrm{~g}$ test bread that had to be consumed by the participants contained $20 \mathrm{~g}$ PR. The control bread contained no PR, but was otherwise identical to the test bread. Here too, an amount of $250 \mathrm{~g} / \mathrm{d}$ had to be ingested. The bread was made using wheat flour, rye flour, crushed rye grain, oat and flax seeds. The total tocopherol concentrations in the control and test bread were 3.86 and $6.32 \mu \mathrm{mol} / 100 \mathrm{~g}$, respectively. The sum of inositol hexa-, penta-, tetra- and triphosphate concentration was $0.50 \mathrm{mmol} / 100 \mathrm{~g}$ for control bread and $0.89 \mathrm{mmol} / 100 \mathrm{~g}$ for test bread.
The intervention study comprised three periods: a $5 \mathrm{~d}$ baseline period consisting of a normal diet without intervention (period 1; PI) for obtaining baseline data. It was followed by a 4 -week period with an intake of control bread (period 2; PII) which was then substituted by test bread in the next 4-week intervention period (period 3; PIII). During baseline and the last $5 \mathrm{~d}$ of PII and PIII a standardised diet was administered. At the same time, complete stool and $24 \mathrm{~h}$ urine were collected for $3 \mathrm{~d}$ as well as one blood sample that was taken by authorised nurses in the morning after overnight fasting. During the standardised diets the consumption of the respective bread was continued. Participants were instructed to eat normally, not to go on a diet and to try to include the bread in their eating habits without increasing their daily energy intake during the study. Except for the control and test bread in the respective periods, the $5 \mathrm{~d}$ standardised diet packages were identical between the three study periods and daily included one warm meal, fruits, vegetables, dairy products, bread-spreads and drinks. Participants were free to consume everything provided in the food package, though they were advised to resemble their food intake at each standardised diet period. The uneaten daily portions had to be returned for quantification of the actual food consumption.

If not described differently, nutrient intake was calculated using PRODI ${ }^{\circledR} 5.4$ software (Wissenschaftliche Verlagsgesellschaft $\mathrm{mbH}$, Stuttgart, Germany). At the start and the end of the study, a bioelectrical impedance analysis (BIA) was conducted for purposes of monitoring the physical constitution of the participants (BIA 2000-C; Data Input, Darmstadt, Germany).

\section{Subjects}

Initially, we recruited twenty voluntary female smokers and twenty female non-smokers via placards and newspaper advertisements. Subsequently, four participants from the group of smokers dropped out of the study for individual reasons (for example, illness, lack of time). Thus, results presented are based on a total of thirty-six participants; twenty non-smokers and sixteen smokers. Smokers were defined by a consumption of at least five cigarettes/d. The mean cigarette consumption was 9 (SD 5; range 3-20) cigarettes/d. Participants were allowed to smoke before blood withdrawals. Moreover, twenty-two participants (ten smokers and twelve non-smokers) used a hormonal contraceptive, whereas fourteen participants (six smokers, eight non-smokers) did not. Criteria for including subjects into the study were not doing serious sports, no diagnosed diseases, and no use of nutritional supplements 2 weeks before and during the study. Participants were aged 24 (SD 3; range18-33) years. The mean BMI constituted 23.3 (SD 3.7; range 17.9$32 \cdot 8) \mathrm{kg} / \mathrm{m}^{2}$ with a body size of 170 (SD 7; range $\left.157-186\right) \mathrm{cm}$ and a body weight of 67 (SD 11; range 53-97) kg. All test individuals were informed as regards the purpose, the course and their responsibilities during the study before they gave their informed written consent. The study was approved by the Ethics Committee of the Medical Faculty of the Friedrich Schiller University of Jena, Germany (approval no. 1485-01/05).

\section{Preparation of human samples}

The complete, fresh stool was collected in plastic bags, transported to the laboratory and immediately stored at $-20^{\circ} \mathrm{C}$. 
After receiving all stool samples, stool was defrosted, homogenised and subsequently lyophilised, separately for each participant and period. For gaining faecal water, samples of the homogenised, defrosted stool were weighed into polyethylene tubes (Beckman Coulter, Munich, Germany), and centrifuged for $4 \mathrm{~h}$ at $21000 \mathrm{rpm}$ and $4^{\circ} \mathrm{C}$ (Beckman J2-21). The supernatant fraction representing the faecal water was stored in cryogenic tubes (Roth, Karlsruhe, Germany) at $-20^{\circ} \mathrm{C}$. Samples of urine taken from the daily samples were stored at $-20^{\circ} \mathrm{C}$. The defrosted samples were mixed according to the proportion of the daily excreted urine volume, separately for each participant and period. Blood was collected into serum tubes (BD Vacutainer Systems, Heidelberg, Germany) and centrifuged for $20 \mathrm{~min}$ at $4000 \mathrm{rpm}$. Serum obtained was frozen at $-20^{\circ} \mathrm{C}$ until analysis.

\section{Tocopherols}

According to Kuhnt et al. lyophilised food and stool were added with ascorbic acid (Fluka, Buchs, Switzerland) and saponified $^{(25)}$. Extraction was carried out using $n$-hexane containing 2,6-di-tert-butyl-p-kresol (BHT) (VWR, Leuven, Belgium and Fluka, Buchs, Switzerland, respectively). With each batch executed, a reference milk powder of defined tocopherol concentration was analysed in parallel (BCR-421, Report EUR 18320 EN; Promochem, Wesel, Germany). Serum samples were prepared with ethanol-BHT solution (ethanol: Roth, Karlsruhe, Germany), shaken and extracted using $n$-hexane containing BHT. Extracts were measured by means of HPLC-fluorescence (Shimadzu, Tokyo, Japan; Nucleosil $100 \mathrm{NH}_{2}$ column, $250 \times 4 \mathrm{~mm}$; Macherey \& Nagel, Dueren, Germany). Analysed $\alpha-, \beta-, \gamma-$ and $\delta$-tocopherol concentrations were summed up to total tocopherol (tocopherol standards; Calbiochem, Darmstadt, Germany).

\section{Hydroxylation products of salicylic acid in stool (antioxidant capacity of stool)}

According to the method of Owen et al. phosphate buffer (100 mM, $\mathrm{KH}_{2} \mathrm{PO}_{4}$ and $\mathrm{K}_{2} \mathrm{HPO}_{4}$; Merck, Darmstadt, Germany) was prepared with EDTA $(500 \mu \mathrm{M}$; Roth, Karlsruhe, Germany), $\mathrm{FeCl}_{3}$ (iron $50 \mu \mathrm{M}$; Merck, Darmstadt, Germany) and salicylic acid (2 mM; Merck, Darmstadt, Germany) in HPLC-grade water $(\mathrm{pH} 6 \cdot 5)^{(14)} \cdot \mathrm{Fe}^{3+}$ and EDTA are added to the test system to support the generation of hydroxyl radicals. These radicals oxidise the salicylic acid to 2,3- and 2,5-dihydroxy benzoic acid (DHBA) and catechol which can all be quantified. Lyophilised stool $(0 \cdot 1 \mathrm{~g})$ was mixed with $10 \mathrm{ml}$ phosphate buffer and incubated in an orbital shaker at $200 \mathrm{rpm}$ for $18 \mathrm{~h}$ at $37^{\circ} \mathrm{C}$. After centrifugation $(4000 \mathrm{rpm}$, 40 min, Rotina 46; Hettich Zentrifugen, Tuttlingen, Germany), the supernatant fraction was filtered using a sterile, pyrogenfree filter $(0 \cdot 2 \mu \mathrm{m}$, Chromafil; Macherey \& Nagel, Dueren, Germany) and measured by means of HPLC-UV (diphenols $325 \mathrm{~nm}$, catechol $278 \mathrm{~nm}$; column: Hypersil C18 ODS II, $250 \times 4 \mathrm{~mm}$; Agilent, Waldbronn, Germany). Unlike Owen et al. for gradient elution the mobile phase consisted of methanol (VWR, Leuven, Belgium) and ammonium acetate buffer (Merck, Darmstadt, Germany; pH 3.6 with acetic acid, Roth, Karlsruhe, Germany) ${ }^{(14)}$. Standard curves obtained from catechol, 2,5-DHBA and 2,3-DHBA (Sigma-Aldrich, Steinheim, Germany) were utilised for calculating the results.

\section{Iron parameters}

For the determination of serum Fe parameters, the Abbott Architect c8000 analyser and the corresponding test kits were used according to the manufacturer's instructions (Abbott, Wiesbaden, Germany and Abbott Laboratories, Baar, Switzerland). The ferritin assay was performed with the chemiluminescent microparticle immunoassay. Serum Fe was analysed colorimetrically by means of the Fe assay; the transferrin was analysed using an immunoturbidimetric assay.

$\mathrm{Fe}$ concentrations in test and control bread as well as in the stool were analysed via ICP-OES (Spectroflame, Spectro, Kleve, Germany) according to DIN 38406 (E22). The stool sample was reduced to ash and mixed with water and $\mathrm{HCl}$ (Roth, Karlsruhe, Germany), then heated and filtrated. Before measuring, the sample was acidified with ultrapure $\mathrm{HNO}_{3}$ (Roth, Karlsruhe, Germany) to a final concentration of $2 \%$ of the sample and diluted at a ratio of 1:2 with ultrapure water. Unlike described in the used DIN method, the calibration range was adjusted according to the expected concentrations (0.005-5.0 mg/l, multi-element standard Merck IV; Merck, Darmstadt, Germany).

The Fe intake from the foods during the standardised diet that were consumed besides the breads was calculated using the PRODI ${ }^{\circledR} 5.4$ software (Wissenschaftliche Verlagsgesellschaft $\mathrm{mbH})$.

\section{Cyto- and genotoxicity of faecal water}

Cyto- and genotoxicity of faecal water were tested using HT29 clone cells treated with $10 \%$ faecal water for $30 \mathrm{~min}$ at $37^{\circ} \mathrm{C}$. The determination of cell viability before and after incubation with faecal water was accomplished via the Trypan Blue exclusion $^{(26)}$. Analyses on genotoxicity of the faecal water were performed using single-cell micro-gel-electrophoresis (comet assay) measuring tail intensity according to Oberreuther-Moschner et al. ${ }^{(27)}$.

\section{Urinary 8-oxo-2'-deoxyguanosine}

The urine samples were purified on a C18 EC column (Macherey \& Nagel, Dueren, Germany). 8-OxodG was eluted with methanol (VWR, Leuven, Belgium), concentrated under a stream of $\mathrm{N}_{2}$, resolved in HPLC-grade water and measured by means of HPLC-electrochemical detection (Shimadzu, Tokyo, Japan; column: Hypersil C18 ODS II, $250 \times 4 \mathrm{~mm}$; Agilent, Waldbronn, Germany) according to Kuhnt et al. ${ }^{(25)}$. For calibration purposes, 8-oxodG was purchased from Sigma-Aldrich (Munich, Germany).

\section{$F_{2}$-isoprostanes and prostaglandin $F_{2 \alpha}$ metabolite}

8-Iso-PGF $2 \alpha$, a standard marker of oxidative stress, and 15-keto-dihydro- $\mathrm{PGF}_{2 \alpha}$, a reliable marker of inflammatory response formed through the cyclo-oxygenase pathway, were analysed in urine samples using two separate RIA, as described previously ${ }^{(28,29)}$. 


\section{Statistical analysis}

Statistical analysis was carried out using the SPSS 14.0 software package (SPSS, Inc., Chicago, IL, USA). Results were tested for normal distribution by means of the KolmogorovSmirnov test. If not described differently, significance was checked using repeated-measures ANOVA. The one-sided Mann-Whitney $U$ test was used to compare subgroups. For correlation analysis, the Pearson's correlation coefficient was determined. Values were referred to as significant at $P<0 \cdot 05$.

\section{Results}

The physical constitution of subjects measured assessed via bioelectrical impedance analysis remained unchanged during the study. The mean energy intake with the standardised diets was 7350 (SD 811) kJ/d (baseline). This value significantly increased during control (8000 (SD 1063) kJ/d) and test bread consumption (7771 (SD 1185) kJ/d; $P<0 \cdot 05)$. The results were only split into subgroups of smokers, non-smokers as well as users and non-users of hormonal contraceptives when regarded as necessary, i.e. if there were any significant changes apparent between the periods.

\section{Tocopherols}

The total tocopherol intake at baseline, control and intervention was 42.0 (SD 5.9), 43.3 (SD 6.7) and 51.4 (SD 8.2) $\mu \mathrm{mol} / \mathrm{d}$, respectively $(P<0 \cdot 001)$. The total tocopherol intake was the same for smokers and non-smokers.

Faecal total tocopherol excretion was significantly increased from control to intervention $(P=0.046)$ (Table 1). With respect to the subgroups, the total tocopherol excretion in smokers significantly increased from baseline and control to intervention $(P<0.05)$. At intervention, excretion significantly differed between smokers and non-smokers $(P=0 \cdot 008)$.

For the whole study group, lipid-adjusted total tocopherol concentration in serum was significantly decreased at control compared with baseline and intervention period $(P<0 \cdot 01)$. Similar relationships between the periods were present in non-smokers $(P<0.05)$ and in smokers, though in smokers the decrease from baseline to control was a tendency only $(P=0 \cdot 057)$.

\section{Diphenols in stool}

Although the analyses were carried out on lyophilised stool samples, the results were extrapolated to the daily excreted amount of fresh matter, because of the increased daily stool mass and DM (D Helbig, unpublished results) at intervention (Table 2). The generation of the diphenols 2,3- and 2,5-DHBA in faeces was significantly increased from baseline to intervention $(P<0 \cdot 01)$. The subgroup of non-smokers showed no changes regarding the diphenols, whereas in smokers concentrations during intervention increased compared with baseline and control $(P<0 \cdot 05)$.

Blind tests (without faecal samples), tests with additional antioxidants (gallic acid, trolox; $0.04 \mathrm{M}$ being the tenfold of the expected molarity of each diphenol) and tests without $\mathrm{Fe}$ and EDTA were also conducted. Diphenols were not detected in any of the incubation batches. The salicylic acid peak in the faecal chromatogram confirmed that salicylic acid was present in abundance. In contrast, catechol could not be detected. The correlation determined between total DHBA (based on $100 \mathrm{mg}$ fresh matter) and 8-oxodG was $r$ 0.328 during PI $(P=0.051)$, $r 0.354$ during PII $(P=0.034)$ and $r 0.324$ during PIII $(P=0 \cdot 054)$. Further, total DHBA (extrapolated to daily excreted fresh matter) and faecal Fe excretion were highly correlated $(r 0.707$ for PI $(P<0.001), r 0.568$ for PII $(P<0.001)$ and $r 0.744$ for PIII $(P<0.001))$. Total DHBA (extrapolated to daily excreted fresh matter) and faecal water genotoxicity were negatively associated $(r-0.273$ for PI $(P=0.107), r-0.313$ for PII $(P=0.063)$ and $r-0.134$ for PIII $(P=0 \cdot 434))$.

\section{Iron parameters}

Fe intake increased steadily from baseline to control and again to intervention $(P<0 \cdot 001)$ (Table 3). Similarly, Fe excretion increased with control and test bread $v$. baseline $(P<0 \cdot 01)$. However, serum ferritin concentration was significantly lower at intervention than at baseline $(P<0 \cdot 05)$. Excluding

Table 1. Changes in serum and stool total tocopherol concentrations after control bread consumption and intervention with blackcurrant seed press residue-enriched test bread considering smoking habits

(Mean values and standard deviations)

\begin{tabular}{|c|c|c|c|c|c|c|}
\hline & \multicolumn{2}{|c|}{ Baseline } & \multicolumn{2}{|c|}{ Control bread } & \multicolumn{2}{|c|}{ Test bread } \\
\hline & Mean & SD & Mean & SD & Mean & SD \\
\hline \multicolumn{7}{|c|}{ Serum $(\mu \mathrm{mol} / \mathrm{mmol}$ serum lipids) $\dagger$} \\
\hline All $(n 36)$ & $5 \cdot 43^{\mathrm{a}}$ & 1.39 & $4.34^{\mathrm{b}}$ & 1.79 & $5.62^{a}$ & 1.23 \\
\hline Smokers (n 16) & $5 \cdot 11^{\mathrm{a}, \mathrm{b}}$ & 1.35 & $3.87^{\mathrm{a}}$ & 1.76 & $5 \cdot 54^{\mathrm{b}}$ & 1.32 \\
\hline Non-smokers $(n 20)$ & $5 \cdot 69^{\mathrm{a}}$ & 1.40 & $4.72^{\mathrm{b}}$ & 1.77 & $5.68^{\mathrm{a}}$ & $1 \cdot 18$ \\
\hline \multicolumn{7}{|l|}{ Stool $(\mu \mathrm{mol} / \mathrm{d})$} \\
\hline All $(n$ 36)‡ & $23 \cdot 7^{a}$ & 14.9 & $26 \cdot 2^{a, b}$ & $19 \cdot 7$ & $28 \cdot 1^{\mathrm{b}}$ & $17 \cdot 8$ \\
\hline Smokers $(n 16)$ & $27 \cdot 5^{a}$ & $18 \cdot 6$ & $26 \cdot 9^{a}$ & $17 \cdot 4$ & $36 \cdot 9^{b *}$ & $20 \cdot 3$ \\
\hline Non-smokers $(n 20)$ & $20 \cdot 6$ & $10 \cdot 7$ & $25 \cdot 7$ & $21 \cdot 8$ & $21 \cdot 1$ & $11 \cdot 8$ \\
\hline
\end{tabular}

a,b,c Mean values within a row with unlike superscript letters were significantly different $(P \leq 0.05)$.

${ }^{*}$ Mean value was significantly different from that of the non-smokers $(P \leq 0.05)$.

†Tocopherols were adjusted to serum lipids (TAG + cholesterol).

$\ddagger$ Values were not distributed normally; significance was calculated by means of the Wilcoxon test. 
Table 2. Concentration (mmol/d fresh matter) of 2,3- and 2,5-dihydroxy benzoic acid (DHBA) in stool, generated from salicylic acid in vitro after control bread consumption and intervention with blackcurrant seed press residue-enriched test bread considering smoking habits (Mean values and standard deviations)

\begin{tabular}{|c|c|c|c|c|c|c|}
\hline & \multicolumn{2}{|c|}{ Baseline } & \multicolumn{2}{|c|}{ Control bread } & \multicolumn{2}{|c|}{ Test bread } \\
\hline & Mean & SD & Mean & SD & Mean & SD \\
\hline \multicolumn{7}{|l|}{ All $(n 36)$} \\
\hline 2,3-DHBA† & $199^{a *}$ & 131 & $220^{a, b}$ & 130 & $271^{b *}$ & 197 \\
\hline 2,5-DHBA & $107^{\mathrm{a}}$ & 74 & $125^{a, b}$ & 82 & $156^{\mathrm{b}}$ & 113 \\
\hline Total DHBA & $306^{a}$ & 201 & $344^{a, b}$ & 211 & $426^{\mathrm{b} *}$ & 308 \\
\hline \multicolumn{7}{|l|}{ Smokers ( $n 16)$} \\
\hline 2,3-DHBA & $229^{a}$ & 163 & $236^{a, c}$ & 91 & $373^{\mathrm{b}}$ & 246 \\
\hline 2,5-DHBA & $123^{\mathrm{a}}$ & 85 & $132^{\mathrm{a}, \mathrm{c}}$ & 51 & $213^{\mathrm{b}}$ & 136 \\
\hline Total DHBA & $351^{a}$ & 242 & $368^{a}$ & 139 & $586^{\mathrm{b}}$ & 379 \\
\hline \multicolumn{7}{|c|}{ Non-smokers $(n 20)$} \\
\hline 2,3-DHBA & 175 & 96 & 206 & 155 & 189 & 89 \\
\hline 2,5-DHBA & 94 & 63 & 120 & 102 & 110 & 63 \\
\hline Total DHBA & 270 & 157 & 324 & 257 & 299 & 151 \\
\hline \multicolumn{7}{|c|}{$P$ between smoking habits } \\
\hline 2,3-DHBA & NS & & NS & & 0.010 & \\
\hline 2,5-DHBA & NS & & NS & & 0.007 & \\
\hline Total DHBA & NS & & NS & & 0.011 & \\
\hline
\end{tabular}

a,b,c Mean values within a row with unlike superscript letters were significantly different $(P \leq 0.05)$

* Mean value was marginally significantly different from that for the control bread $(P \leq 0 \cdot 1)$.

$\dagger$ Values were not distributed normally; significance was calculated by means of the Wilcoxon test.

anaemic participants, ferritin concentrations were $27 \cdot 0$ (SD 17.2) $\mu \mathrm{g} / \mathrm{l}$ (PI, $n$ 31), $27 \cdot 8$ (SD 16.1) $\mu \mathrm{g} / \mathrm{l}$ (PII, $n$ 26) and 22.2 (SD 11.6) $\mu \mathrm{g} / 1$ (PIII, $n$ 30) with $P<0.05$ for both, baseline and control compared with intervention (Wilcoxon test).

\section{Cyto- and genotoxicity of faecal water}

To evaluate genotoxicity, HT29 clone cell viability was assessed before and after incubation with faecal water. Viability at intervention was significantly reduced compared with baseline and control $(P<0.05)$ (Table 4). In non-smokers, cell viability was significantly decreased from baseline to intervention $(P<0.001)$. There were no changes for smokers and non-smokers between the control and intervention periods. After cell incubation with faecal water, tail intensity significantly increased during intervention compared with both baseline and control $(P<0.05)$. In smokers, no changes occurred throughout the study periods. In non-smokers, the tail intensity increased with intervention $v$. baseline period $(P<0 \cdot 05)$. Cell viability and genotoxicity of faecal water correlated negatively at the control and intervention periods (PI: $r-0.208(P=0.228)$; PII: $r-0.764(P<0.001)$; PIII: $r-0.301(P=0.075))$.

\section{Urinary 8-oxo-2'-deoxyguanosine}

The daily urinary 8-oxodG excretion was not affected by the control bread compared with baseline, both in the whole study population and in the subgroups of smokers and nonsmokers (Table 4). There was a tendency in the daily excretion towards an increase for the whole study population after test bread compared with control $(P<0 \cdot 1)$ and it was significantly increased in non-smokers $(P<0.05)$. There were no significant changes between the periods within the subgroups of users and non-users of hormonal contraceptives, although the 8-oxodG excretion at intervention was significantly higher in the nonusers $(1.21 \mathrm{nmol} / \mathrm{kg}$ per $24 \mathrm{~h})$ compared with the users $(0.76 \mathrm{nmol} / \mathrm{kg}$ per $24 \mathrm{~h})(P=0 \cdot 004)$.

\section{$F_{2}$-isoprostanes and prostaglandin $F_{2 \alpha}$ metabolite}

Urinary 8 -iso- $\mathrm{PGF}_{2 \alpha}$ and 15-keto-dihydro- $\mathrm{PGF}_{2 \alpha}$ were only analysed in samples collected from the control and intervention periods to follow both oxidative stress and the inflammatory response (Table 5). No significant changes were found for the whole study population, and for the smokers and non-smokers separated. Interestingly, the excretion of urinary 8 -iso-PGF $2 \alpha$ in volunteers using no hormonal contraceptive was significantly increased by the intervention.

\section{Discussion}

The consumption of bread enriched with blackcurrant PR produced effects on biomarkers related to oxidative stress via different PR components, for example, phytic acid, PUFA or Fe. The decreased serum total tocopherol achieved with control bread compared with baseline was possibly a result of replacing the

Table 3. Iron intake, iron excretion, and iron serum parameters after control bread consumption and intervention with blackcurrant seed press residue-enriched test bread (Mean values and standard deviations for thirty-six subjects)

\begin{tabular}{|c|c|c|c|c|c|c|}
\hline & \multicolumn{2}{|c|}{ Baseline } & \multicolumn{2}{|c|}{ Control bread } & \multicolumn{2}{|c|}{ Test bread } \\
\hline & Mean & SD & Mean & SD & Mean & SD \\
\hline Intake $(\mathrm{mg} / \mathrm{d}) \dagger$ & $10 \cdot 4^{\mathrm{a}}$ & $1 \cdot 1$ & $12 \cdot 0^{\mathrm{b}}$ & 1.4 & $12 \cdot 8^{c}$ & 1.5 \\
\hline Faecal excretion (mg/d) & $6 \cdot 37^{\mathrm{a}}$ & $3 \cdot 60$ & $8 \cdot 24^{b}$ & 5.08 & $9 \cdot 52^{b}$ & $5 \cdot 14$ \\
\hline $\mathrm{Fe}$ in serum $(\mathrm{mg} / \mathrm{l})$ & 1.09 & 0.41 & $1 \cdot 17$ & 0.45 & 1.07 & 0.45 \\
\hline Ferritin $(\mu \mathrm{g} / \mathrm{l}) \dagger$ & $23 \cdot 9^{a}$ & $17 \cdot 7$ & $21 \cdot 6^{a, b}$ & $17 \cdot 0$ & $19 \cdot 2^{b}$ & $12 \cdot 7$ \\
\hline Transferrin $(\mathrm{g} / \mathrm{l})$ & $3 \cdot 08^{*}$ & 0.65 & $3 \cdot 20$ & 0.64 & $3.09^{*}$ & 0.63 \\
\hline Transferrin saturation (\%) & $26 \cdot 4$ & 11.4 & $27 \cdot 3$ & 11.4 & $25 \cdot 8$ & $11 \cdot 6$ \\
\hline
\end{tabular}

\footnotetext{
${ }^{a, b, c}$ Mean values within a row with unlike superscript letters were significantly different $(P \leq 0.05)$. Results without superscripts in a row had no significant differences.

${ }^{*}$ Mean value was marginally significantly different from that for the control bread $(P \leq 0 \cdot 1)$.

†Intake achieved using PRODI ${ }^{\circledR} 5.4$ software (Wissenschaftliche Verlagsgesellschaft mbH, Stuttgart, Germany).
} 
Table 4. Cyto- and genotoxicity of faecal water (comet assay, given in fluorescence tail intensity) and 8-oxo-2'-deoxyguanosine (8-oxodG) in urine after control bread consumption and intervention with blackcurrant seed press residue-enriched test bread considering smoking habits

(Mean values and standard deviations)

\begin{tabular}{|c|c|c|c|c|c|c|}
\hline & \multicolumn{2}{|c|}{ Baseline } & \multicolumn{2}{|c|}{ Control bread } & \multicolumn{2}{|c|}{ Test bread } \\
\hline & Mean & SD & Mean & SD & Mean & SD \\
\hline \multicolumn{7}{|l|}{ Cell viability (\%) } \\
\hline All $(n 36) \dagger$ & $98 \cdot 8^{\mathrm{a} *}$ & 1.3 & $95 \cdot 1^{\mathrm{a}}$ & $15 \cdot 8$ & $92 \cdot 5^{\mathrm{b}}$ & $17 \cdot 0$ \\
\hline Smokers $(n 16)$ & $98 \cdot 8$ & 1.2 & $97 \cdot 8$ & $3 \cdot 1$ & $90 \cdot 1$ & $23 \cdot 0$ \\
\hline Non-smokers $(n 20)^{\star}$ & $98 \cdot 7^{a}$ & 1.4 & $92 \cdot 9^{a, b}$ & 21.0 & $94 \cdot 5^{\mathrm{b}}$ & $10 \cdot 3$ \\
\hline \multicolumn{7}{|l|}{ Comet assay (\%) } \\
\hline All $(n 36)$ & $13 \cdot 5^{\mathrm{a}}$ & 8.4 & $13 \cdot 9^{a}$ & 9.9 & $17 \cdot 2^{b}$ & 13.4 \\
\hline Smokers $(n 16)$ & $14 \cdot 2$ & $9 \cdot 0$ & $12 \cdot 9$ & 7.4 & $15 \cdot 0$ & $9 \cdot 1$ \\
\hline Non-smokers ( $n$ 20) & $13 \cdot 0^{\mathrm{a}}$ & $8 \cdot 2$ & $14 \cdot 7^{\mathrm{a}, \mathrm{b}}$ & 11.7 & $19 \cdot 1^{\mathrm{b} *}$ & $16 \cdot 1$ \\
\hline \multicolumn{7}{|c|}{ 8-OxodG (nmol/kg per $24 \mathrm{~h})$} \\
\hline All $(n 36) \dagger$ & 0.749 & 0.684 & 0.723 & 0.823 & $0.935^{\star}$ & 1.236 \\
\hline Smokers $(n 16)$ & 0.805 & 0.845 & 0.693 & 0.829 & 0.889 & 1.624 \\
\hline Non-smokers ( $n$ 20) & $0.705^{\mathrm{a}, \mathrm{b}}$ & 0.541 & $0.747^{\mathrm{a}}$ & 0.838 & $0.971^{b}$ & 0.853 \\
\hline
\end{tabular}

${ }^{\mathrm{a}, \mathrm{b}}$ Mean values within a row with unlike superscript letters were significantly different $(P \leq 0.05)$.

* Mean value was marginally significantly different from that for the control bread $(P \leq 0.1)$.

$\dagger$ Values were not distributed normally; significance was calculated by means of the Wilcoxon test.

normally consumed foods in favour of the control bread. Though tocopherol intake was increased with the control bread, suggesting an increase of serum tocopherol, single isomers may have been less absorbable, resulting in a decrease. This effect was reduced by the PR intake at intervention and serum total tocopherol concentration increased compared with the control bread. Serum total tocopherol concentrations showed no differences between smokers and non-smokers at any period, confirming the findings of Kontush et al. ${ }^{(7)}$.

Blackcurrant berries and seeds contain significant quantities of proanthocyanidins ${ }^{(30,31)}$. Because dietary polyphenols are virtually all degraded to monomers by normal gut bacteria, the phenol concentration in faecal water is high enough to bring about physiological changes in the stool ${ }^{(32)}$. Due to low absorption rates of polyphenols, effects in the gastrointestinal tract might be even higher than in the body itself. Cyanidin-3-glycoside, a chief component of blackcurrant anthocyanins, was protective towards $\mathrm{H}_{2} \mathrm{O}_{2}$-induced DNA strand breaks in colonocytes in vitro, but failed at physiological dose rates in rats in vivo ${ }^{(31,33)}$. Further, phytic acid from bread and also from PR might improve the antioxidant properties of both the breads.
Contrary to results achieved from studies on polyphenols and phytic acid, the combination of bread and PR increased the formation of diphenols compared with control bread, implying a decreased capacity in the scavenging of hydroxyl radicals with the consumption of the PR bread. Generation of the diphenols 2,3- and 2,5-DHBA remained unaffected by the bread itself. Smoking increased the generation of both diphenols at intervention compared with control and baseline. Thus, the faeces from smokers showed a reduced antioxidant capacity when PR bread was consumed. In addition to the polyphenols, the consumption of tocopherols should also point to an increase in the faecal antioxidant capacity rather than a decrease. However, it has been reported that tocopherols do not effectively scavenge the ubiquitously occurring hydroxyl radicals ${ }^{(34)}$.

An analysis of the components of blackcurrant PR exhibited the presence of significant amounts of $\mathrm{Fe}$ in the $\mathrm{PR}^{(1)}$. Therefore, the Fe parameters were also taken into consideration in this human intervention study. Most of the PR Fe probably came from the mill during crushing. Steel dust is a catalyst for Fenton-like oxidations ${ }^{(35)}$. The increased $\mathrm{Fe}$ intake was not reflected in an improved $\mathrm{Fe}$ status of the

Table 5. 8-Iso-PGF $2 \alpha$ and 15-keto-dihydro- $\mathrm{PGF}_{2 \alpha}$ excretion (nmol/24h) in urine after control bread consumption and intervention with blackcurrant seed press residue-enriched test bread considering smoking habits and the use of hormonal contraceptives (Mean values and standard deviations)

\begin{tabular}{|c|c|c|c|c|c|c|c|c|c|c|}
\hline & \multicolumn{4}{|c|}{ 8-Iso-PGF $2 \alpha$} & \multicolumn{5}{|c|}{ 15-Keto-dihydro-PGF $2 \alpha$} & \multirow[b]{3}{*}{$P^{*}$} \\
\hline & \multicolumn{2}{|c|}{ Control bread } & \multicolumn{2}{|c|}{ Test bread } & \multirow[b]{2}{*}{$P^{*}$} & \multicolumn{2}{|c|}{ Control bread } & \multicolumn{2}{|c|}{ Test bread } & \\
\hline & Mean & SD & Mean & SD & & Mean & SD & Mean & SD & \\
\hline All $(n 36)$ & $9 \cdot 32$ & $3 \cdot 16$ & $9 \cdot 71$ & 2.99 & 0.495 & $2 \cdot 30$ & 0.89 & $2 \cdot 47$ & 0.91 & 0.176 \\
\hline Smokers ( $n$ 16) & $9 \cdot 41$ & $2 \cdot 41$ & $9 \cdot 71$ & $3 \cdot 31$ & 0.590 & $2 \cdot 38$ & $1 \cdot 10$ & $2 \cdot 33$ & 1.00 & 0.428 \\
\hline Non-smokers $(n 20)$ & 8.56 & $2 \cdot 16$ & $9 \cdot 28$ & 2.54 & 0.323 & $2 \cdot 15$ & 0.60 & 2.57 & 0.89 & 0.409 \\
\hline Contraceptive users ( $n$ 22) & $9 \cdot 22$ & $3 \cdot 68$ & $8.73 \dagger$ & $2 \cdot 68$ & 0.518 & $2 \cdot 16$ & $0 \cdot 70$ & $2 \cdot 22 \dagger$ & 0.78 & 0.673 \\
\hline Non-contraceptive users $(n 14)$ & 9.48 & $2 \cdot 22$ & $11 \cdot 26$ & $2 \cdot 87$ & 0.042 & 2.53 & 1.13 & $2 \cdot 87$ & 0.98 & 0.146 \\
\hline
\end{tabular}

*Between control and test bread for each prostane.

$\dagger$ Mean value was significantly different from that for the non-users of hormonal contraceptives $(P \leq 0.05)$. 
participants. In fact, most of the Fe remained in the intestinal lumen, able to act as a damaging factor ${ }^{(36)}$. The concentration of the Fe-storage protein ferritin decreased with consumption of both the control and test bread, indicating an Fe deficiency in the body. An impairment of $\mathrm{Fe}$ status has also been described in women consuming wheat bread ${ }^{(37)}$. The phytic acid in the grain potentially binds faecal $\mathrm{Fe}$ and decreases absorption. The ferritin decrease became even more pronounced with the test bread when excluding the anaemic participants, possibly because PR additionally contained phytic acid. In a prior study with a similar study design, 10 or $20 \mathrm{~g}$ PR were eaten daily in yoghurt for 4 consecutive weeks (D Helbig, unpublished results). Here, ferritin concentrations also decreased significantly from baseline (pure yoghurt) to both intervention periods, suggesting that ferritin decrease is most probably due to the PR.

Phytic acid prevents the generation of hydroxyl radical by chelating $\mathrm{Fe}^{(38)}$. Nevertheless, it appears questionable whether phytic acid has an impact on antioxidant capacity in the in vitro test system. First, phytic acid reduces the hydroxyl radical formation by trapping the $\mathrm{Fe}$, but does not scavenge the hydroxyl radicals produced in the test system. However, phytic acid can have an impact on antioxidant conditions in vivo due to the absence of EDTA ${ }^{(39)}$. Second, when EDTA, which is a stronger Fe scavenger than phytic acid, is added to the test system for in vitro aromatic hydroxylation, the Fe gets detracted from the phytic acid ${ }^{(14)}$. Contrary to phytic acid, EDTA is a promoter of hydroxyl radical generation because of its ability to form a free $\mathrm{Fe}$ coordination site, thus contributing to the oxidation processes $^{(38,40)}$. It is generally assumed that radicals in stool and oxidation of membrane lipids and its chain-reaction products lead to in vivo DNA damage. The correlations determined between DHBA and 8-oxodG and faecal Fe excretion confirm this assumption. The high $\mathrm{Fe}$ content of the digest possibly increased the formation of hydroxyl radicals in vivo, exhausting faecal antioxidants, leading to a reduced antioxidant capacity of the stool. A study using dimethylsulfoxide to scavenge faecal hydroxyl radicals reported that a diet rich in fat and meat and low in fibre showed a thirteen times increased hydroxyl radical production than with a low-fat, vegetarian high-fibre diet ${ }^{(41)}$. In fact, there resulted a $42 \%$ increased faecal $\mathrm{Fe}$ concentration in the high-meat diet. The concentration of faecal $\mathrm{Fe}$ is of no consequence in the test system utilising aromatic hydroxylation, since Fe is added in abundance to provoke the radical generation. However, faecal $\mathrm{Fe}$ might have exhausted faecal antioxidants in the body already.

It was shown that $\mathrm{Fe}^{2+/ 3+}$ also accounts for DNA damage $^{(42)}$. In the present study, faecal Fe concentrations remained unchanged between the control and test breads, but faecal water genotoxicity increased not with the control bread, but only with the test bread. Further, faecal water genotoxicity and faecal Fe concentration did not correlate at test bread consumption, suggesting that increased genotoxicity at intervention was caused by something other than the Fe.

In the present study, faecal water genotoxicity and generated DHBA were negatively associated. Consequently, if both DHBA and faecal water genotoxicity were increased by the intervention, the causes might be attributed to different factors in each case. Further, it has been under discussion that the intake of PUFA promotes DNA damage in the body ${ }^{(43)}$. This is a relevant factor since PR contains high amounts of these fatty acids $(79 \%$ PUFA of total fatty acid methyl esters ${ }^{(1)}$ ) and $4 \mathrm{~g}$ PUFA were ingested daily with the test bread. Heat applied during seed processing and bread baking may have induced lipid peroxidation leading to the increased oxidative DNA damage by the faecal water at PR consumption. No correlations could be found between the genotoxicity of faecal water and the total tocopherol concentration of faeces. Testing on the cell viability supports the results of the comet assay. Cell viability decreased after incubating cells with faecal water from test bread consumption and both parameters correlated negatively at the control and intervention periods. Bile acids, not analysed here, are other possible contributors to elevated reactive oxygen species generation and DNA hydroxylation ${ }^{(44)}$. In addition, fibre has a bile acid-binding capacity ${ }^{(45)}$. Consequently, a change in bile acid concentration and pattern at control and intervention is probably due to increased daily fibre intakes compared with baseline (31\% bread, $54 \%$ bread + PR).

Correlation values of serum total tocopherol or total tocopherol intake in the present study confirm that dietary consumption of antioxidants, particularly of vitamin E, is associated with little or no effects on the urine 8-oxodG concentration $^{(21,33,46)}$. Gackowski et al. described hydroxyl radicals as being the major source of 8 -oxodG formation ${ }^{(47)}$. To attack DNA, these radicals need to be in its immediate vicinity. However, lipophilic vitamins are not usually located near DNA molecules. Non-smokers excreted significantly more 8-oxodG during PR consumption than during intake of control bread. Noticeably, smokers had a two-fold higher standard deviation than non-smokers. Curiously, smokers showed particularly individual reactions to the substances in PR. Generally, 8-oxodG excretion increased with PR bread, although the extent of the increase was influenced by different factors such as smoking habits, the use of hormonal contraceptives, and other, unidentified parameters. There was no correlation between 8-oxodG excretion and faecal water genotoxicity. These results support another study that showed no relationship between faecal water genotoxicity and lymphocyte DNA damage using the comet assay ${ }^{(16)}$. An Fe-dependent increase of 8-oxodG in lymphocytes was found with acute Fe load ${ }^{(48)}$. In the present study, none of the measured serum or faecal $\mathrm{Fe}$ parameters was clearly associated with the urinary 8-oxodG excretion. Thus, the PR component responsible for the increase of urinary 8-oxodG concentration could not be identified.

No differences were shown for prostane excretion in the total study population. However, different outcomes were apparent when looking at the individual subgroups. While hormonal contraceptives obviously protected a PR-induced non-enzymic lipid peroxidation, smoking, contrary to several reports, caused no significant impact ${ }^{(49)}$. There was no association found between prostane excretions and other tested parameters. England et al. found an association between plasma isoprostane 8-epi-PGF $2 \alpha$ and lymphocyte 8-oxodG ${ }^{(50)}$. In the present study, only when urinary 8 -oxodG and prostanes were analysed in the defined subgroups, strong correlations appeared with PR consumption between 8-oxodG and the oxidative stress marker 8 -iso- $\mathrm{PGF}_{2 \alpha}$ excretion for non-smokers and non-users of hormonal contraceptives. Noticeably, these are the subgroups not influenced by special oxidants or antioxidants, in contrast to smokers (increased reactive 
oxygen species) or users of hormonal contraceptives (antioxidant protection). Further, it was shown that a PUFArich diet may increase plasma $\mathrm{PGF}_{2 \alpha}$ concentration ${ }^{(51)}$. Thus, the 8 -iso- $\mathrm{PGF}_{2 \alpha}$ increase might be attributed to the increased PUFA intake with the PR bread.

\section{Conclusion}

Testing complex food matrices makes it difficult to arrive at clear and distinct outcomes compared with single intervention substances. Consequently, effects due to an intervention with berry PR cannot be accredited to one single substance. Nevertheless, the following statements can be presumed: PR consumption resulted in increased faecal $\mathrm{Fe}$ concentrations, decreased faecal antioxidant capacity and increased urine 8-oxodG excretion. The increased genotoxicity of faecal water and partially increased prostane excretion could be due to the higher PUFA intake. PR phytic acid is assumed to account for the decrease in serum ferritin. Furthermore, factors associated with lifestyle, such as smoking habits or the use of hormonal contraceptives, have an impact on the correlations described above. In summary, consuming blackcurrant PR-enriched bread for 4 weeks has adverse effects on the antioxidant status in the body, as serum and stool total tocopherol concentrations were increased. The antioxidant properties of tocopherols had no effect on the measured biomarkers associated with oxidative stress.

\section{Acknowledgements}

We would like to thank the BMBF (Federal Ministry of Education and Research) which provided financial support for this project in the Rephyna ${ }^{\circledR}$ group. We would also like to thank the IGV GmbH Bergholz-Rehbrücke for preparing the test materials, the Fraunhofer IVV Freising for carrying out the microbiological testing, Claudia Nitsch for performing the tocopherol and 8-oxodG analyses and finally the laboratories of Ralf Greiner (Max Rubner-Institut, Federal Research Institute of Nutrition and Food, Karlsruhe, Germany) for operating the inositol phosphate analyses.

D. H. was responsible for the supervision of the human study, sample handling and analyses conducted; A. W. supported tocopherol, 8-oxodG and DHBA analyses; M. G. supported the analyses of cell viability and the performance of the comet assay; S. B. was responsible for the prostane analyses; R. S. was responsible for obtaining funding and supported the statistical evaluation; D. H. and G. J. were responsible for data interpretation; all authors were responsible for critical revision of the manuscript.

None of the authors had any personal or financial conflict of interest.

\section{References}

1. Helbig D, Böhm V, Wagner A, et al. (2008) Berry seed press residues and their valuable ingredients with special regard to black currant seed press residues. Food Chem 111, 1043-1049.

2. Kapasakalidis PG, Rastall RA \& Gordon MH (2006) Extraction of polyphenols from processed black currant (Ribes nigrum L.) residues. J Agric Food Chem 54, 4016-4021.
3. Nakabeppu Y, Sakumi K, Sakamoto K, et al. (2006) Mutagenesis and carcinogenesis caused by the oxidation of nucleic acids. Biol Chem 387, 373-379.

4. Willcox JB, Curb JD \& Rodriguez BL (2008) Antioxidants in cardiovascular health and disease: key lessons from epidemiologic studies. Am J Cardiol 101, Suppl. S75-S86.

5. Kasai H \& Kawai K (2006) Oxidative DNA damage: mechanisms and significance in health and disease. Antioxid Redox Signal 8, 981-983.

6. Öhrvall M, Sundlöf G \& Vessby B (1996) Gamma, but not alpha, tocopherol levels in serum are reduced in coronary heart disease patients. J Intern Med 239, 111-117.

7. Kontush A, Spranger T, Reich A, et al. (1999) Lipophilic antioxidants in blood plasma as markers of atherosclerosis: the role of $\alpha$-carotene and $\gamma$-tocopherol. Atherosclerosis 144, 117-122.

8. Niu X, Zammit V, Upston JM, et al. (1999) Coexistence of oxidized lipids and $\alpha$-tocopherol in all lipoprotein density fractions isolated from advanced human atherosclerotic plaques. Arterioscler Thromb Vasc Biol 19, 1708-1718.

9. Freese R (2006) Markers of oxidative DNA damage in human interventions with fruit and berries. Nutr Cancer 54, 143-147.

10. Wu K, Willett WC, Chan JM, et al. (2002) A prospective study on supplemental vitamin $\mathrm{E}$ intake and risk of colon cancer in women and men. Cancer Epidemiol Biomarkers Prev 11, 1298-1304.

11. Kahle K, Kraus M, Scheppach W, et al. (2006) Studies on apple and blueberry fruit constituents: do the polyphenols reach the colon after ingestion? Mol Nutr Food Res 50, 418-423.

12. Stoner GD, Wang L-S, Zikri N, et al. (2007) Cancer prevention with freeze-dried berries and berry components. Semin Cancer Biol 17, 403-410.

13. Kay CD \& Holub BJ (2002) The effect of wild blueberry (Vaccinium angustifolium) consumption on postprandial serum antioxidant status in human subjects. Br J Nutr 88, 389-397.

14. Owen RW, Spiegelhalder B \& Bartsch H (2000) Generation of reactive oxygen species by the faecal matrix. Gut 46, 225-232.

15. Collins AR (2004) The comet assay for DNA damage and repair. Mol Biotechnol 26, 249-261.

16. Glei M, Habermann N, Osswald K, et al. (2005) Assessment of DNA damage and its modulation by dietary and genetic factors in smokers using the Comet assay: a biomarker model. Biomarkers 10, 203-217.

17. Cooke MS, Rozalski R, Dove R, et al. (2006) Evidence for attenuated cellular 8-oxo-7,8-dihydro-2'-deoxyguanosine removal in cancer patients. Biol Chem 387, 393-400.

18. Cooke MS, Evans MD, Dizdaroglu M, et al. (2003) Oxidative DNA damage: mechanisms, mutation, and disease. FASEB J 17, 1195-1214.

19. Priemé H, Loft S, Klarlund M, et al. (1998) Effect of smoking cessation on oxidative DNA modification estimated by 8-oxo7,8-dihydro-2'-deoxyguanosine excretion. Carcinogenesis 19, $347-351$.

20. Asami S, Hirano T, Yamaguchi R, et al. (1998) Reduction of 8-hydroxyguanine in human leukocyte DNA by physical exercise. Free Radic Res 29, 581-584.

21. Poulsen HE, Loft S, Prieme H, et al. (1998) Oxidative DNA damage in vivo: relationship to age, plasma antioxidants, drug metabolism, glutathione-S-transferase activity and urinary creatinine excretion. Free Radic Res 29, 565-571.

22. Ottaviani JI, Carrasquedo F, Keen CL, et al. (2002) Influence of flavan-3-ols and procyanidins on UVC-mediated formation of 8-oxo-7,8-dihydro-2'-deoxyguanosine in isolated DNA. Arch Biochem Biophys 406, 203-208.

23. Huang H-Y, Appel LJ, Croft KD, et al. (2002) Effects of vitamin $\mathrm{C}$ and vitamin $\mathrm{E}$ on in vivo lipid peroxidation: results of a randomized controlled trial. Am J Clin Nutr 76, 549-555. 
24. Viña J, Sastre J, Pallardó FV, et al. (2006) Role of mitochondrial oxidative stress to explain the different longevity between genders. Protective effect of estrogens. Free Radic Res 40, 1359- 1365 .

25. Kuhnt K, Wagner A, Kraft J, et al. (2006) Dietary supplementation with 11trans- and 12trans-18:1 and oxidative stress in humans. Am J Clin Nutr 84, 981-988.

26. Sandström B (1965) Studies from cells from liver tissue cultivated in vitro. Exp Cell Res 37, 552-568.

27. Oberreuther-Moschner DL, Jahreis G, Rechkemmer G, et al. (2004) Dietary intervention with the probiotics Lactobacillus acidophilus 145 and Bifidobacterium longum 913 modulates the potential of human faecal water to induce damage in HT29clone19A cells. Br J Nutr 91, 925-932.

28. Basu S (1998) Radioimmunoassay of 8-isoprostaglandin $\mathrm{F}_{2 \alpha}$ : an index for oxidative injury via free radical catalysed lipid peroxidation. Prostaglandins Leukot Essent Fatty Acids 58, 319-325.

29. Basu S (1998) Radioimmunoassay of 15-keto-13,14-dihydroprostaglandin $\mathrm{F}_{2 \alpha}$ : an index for inflammation via cyclooxygenase catalysed lipid peroxidation. Prostaglandins Leukot Essent Fatty Acids 58, 347-352.

30. Wu X, Gu L, Prior RL, et al. (2004) Characterization of anthocyanins and proanthocyanidins in some cultivars of ribes, aronia, and sambucus and their antioxidant capacity. J Agric Food Chem 52, 7846-7856.

31. Lu Y \& Foo LY (2003) Polyphenolic constituents of blackcurrant seed residue. Food Chem 80, 71-76.

32. Jenner AM, Rafter J \& Halliwell B (2005) Human fecal water content of phenolics: the extent of colonic exposure to aromatic compounds. Free Radic Biol Med 38, 763-772.

33. Duthie SJ, Gardner PT, Morrice PC, et al. (2005) DNA stability and lipid peroxidation in vitamin E-deficient rats in vivo and colon cells in vitro. Eur J Nutr 44, 195-203.

34. Wang SY \& Jiao H (2000) Scavenging capacity of berry crops on superoxide radicals, hydrogen peroxide, hydroxyl radicals, and singlet oxygen. $J$ Agric Food Chem 48, $5677-5684$.

35. Lee J-M, Kim J-H, Chang Y-Y, et al. (2009) Steel dust catalysis for Fenton-like oxidation of polychlorinated dibenzo-p-dioxins. J Hazard Mater 163, 222-230.

36. Knöbel Y, Weise A, Glei M, et al. (2007) Ferric iron is genotoxic in non-transformed and preneoplastic human colon cells. Food Chem Toxicol 45, 804-811.

37. Bach Kristensen M, Tetens I, Alstrup Jørgensen AB, et al. (2005) A decrease in iron status in young healthy women after long-term daily consumption of the recommended intake of fibre-rich wheat bread. Eur J Nutr 44, 334-340.
38. Graf E, Mahoney JR, Bryant RG, et al. (1984) Iron-catalyzed hydroxyl radical formation. J Biol Chem 259, 3620-3624.

39. Owen RW, Wimonwatwatee T, Spiegelhalder B, et al. (1996) A high performance liquid chromatography system for quantification of hydroxyl radical formation by determination of dihydroxy benzoic acids. Eur J Cancer Prev 5, 233-240.

40. McCord JM \& Day ED Jr (1978) Superoxide-dependent production of hydroxyl radical catalyzed by iron-EDTA complex. FEBS Lett 86, 139-142.

41. Erhardt JG, Lim SS, Bode C, et al. (1997) A diet rich in fat and poor in dietary fiber increases the in vitro formation of reactive oxygen species in human feces. J Nutr 127, 706-709.

42. Rieger MA, Parlesak A, Pool-Zobel BL, et al. (1999) A diet high in fat and meat but low in dietary fibre increases the genotoxic potential of 'faecal water'. Carcinogenesis 20, 2311-2316.

43. Jenkinson AM, Collins AR, Duthie SJ, et al. (1999) The effect of increased intakes of polyunsaturated fatty acids and vitamin $\mathrm{E}$ on DNA damage in human lymphocytes. FASEB $J \mathbf{~ 1 3}$, $2138-2142$.

44. Allgayer H, Kolb M, Stuber V, et al. (2002) Effects of bile acids on base hydroxylation in a model of human colonic mucosal DNA. Cancer Detect Prev 26, 85-89.

45. Buhman KK, Furumoto EJ, Donkin SS, et al. (1998) Dietary psyllium increases fecal bile acid excretion, total steroid excretion and bile acid biosynthesis in rats. $J$ Nutr 128, 1199-1203.

46. Loft S, Vistisen K, Ewertz M, et al. (1992) Oxidative DNA damage estimated by 8-hydroxydeoxyguanosine excretion in humans: influence of smoking, gender and body mass index. Carcinogenesis 13, 2241-2247.

47. Gackowski D, Ciecierski M, Jawieñ A, et al. (2001) Background level of 8-oxo-2'deoxyguanosine in lymphocyte DNA does not correlate with the concentration of antioxidant vitamins in blood plasma. Acta Biochim Pol 48, 535-539.

48. Lucesoli F, Caligiuri M, Roberti MF, et al. (1999) Dose-dependent increase of oxidative damage in the testes of rats subjected to acute iron overload. Arch Biochem Biophys 372, 37-43.

49. Yin H, Gao L, Tai H-H, Murphey LJ, et al. (2007) Urinary prostaglandin $\mathrm{F}_{2 \alpha}$ is generated from the isoprostane pathway and not the cyclooxygenase in humans. J Biol Chem 282, 329-336.

50. England T, Beatty E, Rehman A, et al. (2000) The steadystate levels of oxidative DNA damage and of lipid peroxidation ( $\mathrm{F}_{2}$-isoprostanes) are not correlated in healthy human subjects. Free Radic Res 32, 355-362.

51. Weinberg RB, VanderWerken BS, Anderson RA, et al. (2001) Pro-oxidant effect of vitamin E in cigarette smokers consuming a high polyunsaturated fat diet. Arterioscler Thromb Vasc Biol 21, 1029-1033. 